\title{
Cytotoxic Potentials of Tellurium Nanowires in BALB/3T3 Fibroblast Cells
}

\author{
Sanjeev Kumar Mahto, ${ }^{\dagger}$ T. P. Vinod, ${ }^{\dagger}$ Jinkwon Kim, and Seog Woo Rhee ${ }^{*}$ \\ Green Home Energy Technology Research Center and Department of Chemistry, Kongju National University, \\ Kongju 314-701, Korea. *E-mail: jisanrhee@kongju.ac.kr \\ Received June 13, 2011, Accepted July 29, 2011
}

\begin{abstract}
We have investigated the cytotoxic potential of tellurium (Te) nanowires in BALB/3T3 fibroblast cells. Te nanowires were synthesized through an aqueous phase surfactant assisted method. Toxicological experiments, such as analysis of morphological changes, MTT assay, DAPI staining, and estimation of intracellular reactive oxygen species, were carried out to reveal the cytotoxic effects of Te nanowires. Te nanowires were found to be cytotoxic at all concentrations tested, in a dose-dependent manner. The UV/Vis spectra of Te nanowires suspended in a culture medium showed drastic changes and disappearance of two broad absorption peaks. The physicochemical properties such as, surface charge, size, and shape of Te nanowires were found to be altered during exposure of cells, due to the instability and agglomeration of nanowires in the culture medium. These results suggest that the chemical components of the DMEM medium significantly affect the stability of Te nanowires. In addition, TEM images revealed that necrosis was the basic pattern of cell death, which might stem from the formation of toxic moieties of tellurium, released from nanowire structures, in the bioenvironment. These observations thus suggest that Te nanomaterials may pose potential risks to environmental and human health.
\end{abstract}

Key Words : Tellurium nanowires, Cytotoxicity, Cell morphology, Nuclear structure, Reactive oxygen species

\section{Introduction}

The increasing use of nanomaterials worldwide is gradually increasing likehood of their exposure to the environment and humans. ${ }^{1}$ Because of their unique physical and chemical properties, nanomaterials are rapidly gaining momentum in most sectors of society, including various industrial, biological, and medicinal applications. Alongside these benefits, nanomaterials are also an emerging topic of discussion for their hazards and potential risks to environmental and human health. ${ }^{2}$ Both government organizations and researchers have raised safety concerns over the use of nanomaterials. ${ }^{3,4}$ Therefore, there is an urgent need to address the toxicological effects of all available nanomaterials before using them extensively.

Tellurium (Te) nanomaterials have recently attracted much attention for their unique characteristics and potential applications in photoconductivity, non-linear optical responses, piezoelectricity, and thermoconductivity. ${ }^{5,6}$ Furthermore, Te nanotubes are envisaged to be useful in the delivery of drugs and the protection of biologically active materials (e.g., enzymes or proteins). ${ }^{7}$ This has motivated the rapid development of various types of one-dimensional Te nanostructures, such as nanowires, nanorods, nanotubes, and nanobelts, by a number of research groups. ${ }^{5,6,8,9}$ In addition, due to its high reactivity toward a wealth of chemicals, Te is also being exploited to fabricate other functional materials such as $\mathrm{Bi}_{2} \mathrm{Te}_{3}, \mathrm{CdTe}$, and HgCdTe. ${ }^{10,11}$ However, elemental tellurium is considered to be a non-essential metalloid and hazardous, and its biological and toxicological effects are little understood. ${ }^{12}$ Sodium tellurite was found to be cytotoxic to HeLa cells in a dose-dependent manner. ${ }^{13}$ In addition, tellurite ions induced alteration of erythrocyte membrane. ${ }^{14}$ In another study, diaryl ditellurides showed a meaningful apoptosis induction of HL- 60 cells at doses of $1 \mu \mathrm{M} .{ }^{15}$ However, to the best of our knowledge, the cytotoxicity profile of tellurium nanostructures has yet to be established.

In this work, we investigate the cytotoxic potential of $\mathrm{Te}$ nanowires in BALB/3T3 fibroblast cells. Te nanowires were synthesized through an aqueous phase surfactant assisted method. Nanowires obtained were highly soluble in water, owing to the presence of anionic surfactants on the surface. A fibroblast cell line, BALB/3T3 cells, was chosen because the European Centre of Validation of Alternative Methods (ECVAM) recently considered this in vitro model system for evaluating the cytotoxicity and morphological transformation of chemicals and metal compounds. ${ }^{16,17}$ Also, this cell line has been used to study the toxicology of cobalt nanoparticles. ${ }^{18}$ Toxicological experiments, such as analysis of morphological changes, MTT assay, DAPI staining, and generation of intracellular reactive oxygen species (ROS), were carried out to identify the cytotoxic effects of Te nanowires. Furthermore, TEM images were observed to reveal the mechanism of cell death. Changes to the physicochemical properties and structure of Te nanowires upon exposure to the cell culture medium alone were also studied to elucidate the stability of nanowires in cell culture medium, thereby revealing the probable cause of Te nanowire cytotoxicity.

\footnotetext{
${ }^{\top}$ These authors contributed equally to this work.
} 


\section{Experimental Section}

Preparation and Characterization of Tellurium Nanowires. Dodecylbenzenesulfonic acid, sodium salt (SDBS), poly (sodium 4-styrenesulfonate) (PSS), ammonium sulfide, tellurium powder, sodium sulfite, and sulfur were purchased from Aldrich (MO, USA). Tellurium nanowires were synthesized according to a reported procedure. ${ }^{19}$ Briefly, the tellurium precursor $\left(\mathrm{NH}_{4}\right)_{2} \mathrm{TeS}_{4}$ is reduced with sodium sulfite in the presence of a mixture of surfactants (SDBS and PSS in a molar ratio of 1:1) in an aqueous medium. Te nanowires were obtained after aging the reaction mixture for $30 \mathrm{~h}$. Before using them for a cytotoxicity test, Te nanowires were centrifuged at least 3 times in sterile deionized (DI) water to remove the unbound surfactants.

Scanning electron microscope (SEM) images were obtained from a MIRA LMH (TESCAN) microscope (Czech). $\mathrm{UV} / \mathrm{Vis}$ absorption spectra of the samples were measured using a Scinco S-4100 UV/Vis spectrophotometer (Korea). Zeta potential measurement was performed using a Zetasizer Nano ZS from Malvern Instruments (UK).

Cell Culture and Treatment of Tellurium Nanowires. The mouse embryonic fibroblast cell line BALB/3T3 (Korean Collection for Type Cultures, KCTC) was cultured in low glucose Dulbecco's modified Eagles medium (DMEM, Invitrogen, OR, USA) supplemented with $10 \%$ fetal bovine serum (FBS, Invitrogen, OR, USA) and $1 \%$ penicillin/ streptomycin (P/S, Sigma, MO, USA) in a humidified incubator (Sanyo Electric, Japan) at $37{ }^{\circ} \mathrm{C}$ with $5 \% \mathrm{CO}_{2}$. For the performance of toxicological experiments, cells were cultivated in 12-well tissue culture plates at a seeding density of $4 \times 10^{4}$ cells $/ \mathrm{mL}$. Cells were then treated with culture media containing various concentrations of Te nanowires $(0-125 \mu \mathrm{g} / \mathrm{mL})$ for $48 \mathrm{~h}$ at $60-70 \%$ confluency inside a $\mathrm{CO}_{2}$ incubator. Cells in logarithmic growth phases were used for all the experiments. All treatments were performed in triplicate in three independent experiments.

MTT Assay. The percentage of cell survival was measured using a 3-[4,5-dimethylthiazol-2-yl]-2,5-diphenyltetrazolium (MTT) colorimetric assay. After $48 \mathrm{~h}$ of treatment with Te nanowires, $100 \mu \mathrm{L}$ of $12 \mathrm{mM}$ MTT stock solution (Sigma, MO, USA) dissolved in $1 \mathrm{~mL}$ of culture medium was added to each well of a 12-well plate followed by incubation for $4 \mathrm{~h}$ inside a $\mathrm{CO}_{2}$ incubator at $37{ }^{\circ} \mathrm{C}$. After incubation, the medium was removed and the formed formazan crystals were then dissolved with $2.5 \mathrm{~mL}$ of dimethylsulphoxide (Sigma, MO, USA). The optical density of the formed formazan crystals representative of each well was measured at $540 \mathrm{~nm}$ using a UV/Vis spectrophotometer. At least three independent experiments were performed in each case.

DAPI Staining. After $48 \mathrm{~h}$ of incubation with Te nanowires, cells were fixed in $4 \%$ paraformaldehyde (Aldrich, MO, USA) for $15 \mathrm{~min}$ at $37{ }^{\circ} \mathrm{C}$ followed by washing with phosphate buffered saline (PBS). Subsequently, cells were permeabilized with $0.5 \%$ Triton X-100 (Aldrich, MO, USA) in PBS for $5 \mathrm{~min}$ at $4{ }^{\circ} \mathrm{C}$. Cells were further exposed to
DAPI (4,6-diamidino-2-phenylindole, Molecular Probes, OR, USA) solution in PBS to stain the nuclei. The samples were then viewed by a fluorescence microscope (Olympus IX71, Japan) with an appropriate filter.

Estimation of Intracellular ROS Formation. The intracellular amount of ROS formation was estimated using a fluorescent probe, 2',7'-dichlorofluorescein diacetate (DCFHDA) (Sigma, MO, USA). After exposure to Te nanowires, cells were incubated with a $20 \mu \mathrm{M}$ DCFH-DA probe dissolved in a growth medium for $30 \mathrm{~min}$ at $37^{\circ} \mathrm{C}$. The degree of DCF fluorescence intensity signifies the amount of ROS formed in the cells. The formation of ROS was monitored qualitatively by fluorescence microscopy. The relative fluorescent intensity from the captured images was then quantified using ImageJ software.

Transmission Electron Microscopic (TEM) Analysis of Cells. Cells treated with $125 \mu \mathrm{g} / \mathrm{mL}$ of Te nanowires for $24 \mathrm{~h}$ were fixed in $0.1 \mathrm{M}$ phosphate buffer containing $2.5 \%$ glutaraldehyde $(\mathrm{pH} 7.3)$ for $2 \mathrm{~h}$ at $4{ }^{\circ} \mathrm{C}$. The cells were postfixed in $1 \%$ osmium tetroxide in $0.1 \mathrm{M}$ phosphate buffer on ice for $1 \mathrm{~h}$, dehydrated in an ethanol series, and embedded in Epon 812. Ultra-thin sections were cut on an ultramicrotome (LEICA ULTRACUT-UCT, Germany) with a diamond knife (Diatome, Switzerland), and stained with uranyl acetate for $20 \mathrm{~min}$ followed by lead citrate for $10 \mathrm{~min}$. They were examined by a Tecnai G2 SPIRIT TWIN electron microscope (FEI, USA) at $120-\mathrm{kV}$ accelerating voltage at Korea Basic Science Institute.

Statistical Analysis. Results are presented as mean \pm SD. The statistical significance was determined by a one-way analysis of variance (ANOVA) followed by a Tukey HSD post hoc test. The differences were considered statistically significant when $\mathrm{p}<0.01$. All the treatments were performed in triplicate in three independent experiments.

\section{Results and Discussion}

Te nanomaterials possess various useful properties and have found several industrial applications. However, their eventual widespread use in industry may result in their exposure to an increasing number of workers and consumers. Thus, they may pose potential risks to the environment and human health. In this study, we investigated the cytotoxic effects of tellurium nanowires on BALB/3T3 fibroblast cells.

Alterations in the Morphology and Nuclear Structure of Cells. Using phase contrast microscopy, changes in the morphology of cells treated with different concentrations of Te nanowires were observed. Cells exposed to Te nanowires for $48 \mathrm{~h}$ showed characteristic morphological changes, such as round and oval phenotype, cell shrinkage, and loss of cell adhesion in a dose-dependent manner. These types of morphological changes were not observed in the case of control cells (Figure 1(a)).

Similarly, nuclear structures were observed after staining with DAPI using fluorescence microscopy. Most stained nuclei in controls were healthy and morphologically oval- 
(a)

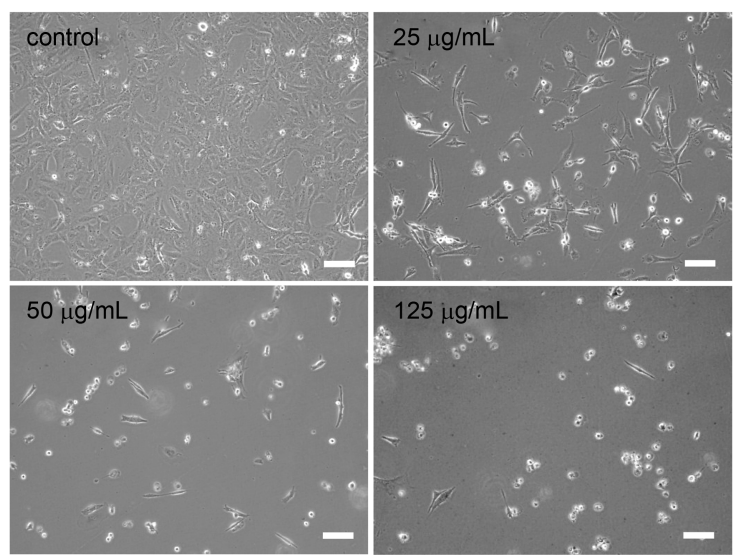

(b)

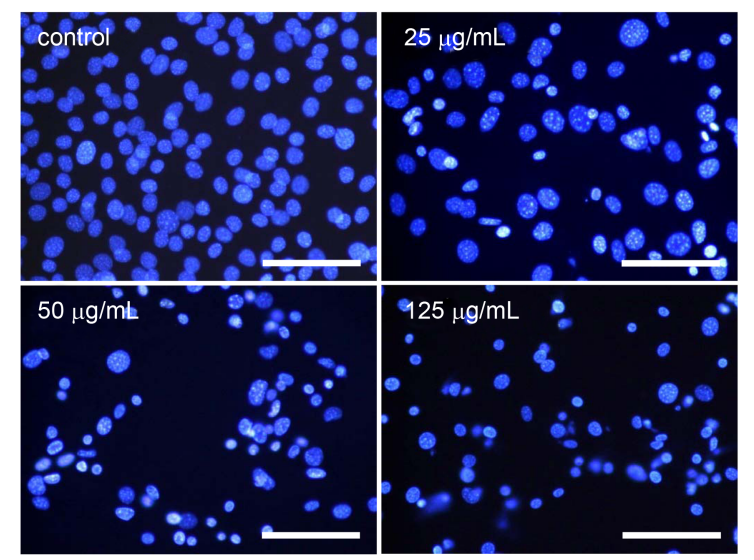

Figure 1. Changes in the morphology of (a) cells and (b) nuclear structure after incubation with Te nanowires for $48 \mathrm{~h}$. The phase-contrast images show characteristic morphological changes in cells, such as round and oval phenotype, cell shrinkage, and loss of cell adhesion in a dose-dependent manner. Similarly, the fluorescent images show highly shrunken and irregularly shaped nuclei in a dose-dependent manner. Scale bars: $100 \mu \mathrm{m}$.

shaped with a diffuse chromatin distribution. In contrast, nuclei of cells treated with Te nanowires for $48 \mathrm{~h}$ exhibited significant changes in their morphology, showing, for example, highly shrunken and irregularly shaped morphological characteristics. These significant changes in nuclear structures were observed in a dose-dependent fashion (Figure 1(b)).

We have also assessed the effects of SDBS and PSS, respectively, on cell morphology. There were no cytotoxic effects of SDBS observed up to concentrations of $50 \mu \mathrm{g} / \mathrm{mL}$ after $48 \mathrm{~h}$ of incubation. However, SDBS showed severe cytotoxic effects such as cell detachment and rounding at a higher concentration of $100 \mu \mathrm{g} / \mathrm{mL}$ (Figure 2(a)). In contrast, PSS was highly cytocompatible at all concentrations (up to $100 \mu \mathrm{g} / \mathrm{mL}$ ) tested (Figure 2(b)).

Dose-dependent Cell Viability. We used a MTT assay to estimate the viability of fibroblast cells under different doses of Te nanowire exposure for $48 \mathrm{~h}$. The results indicated that Te nanowires significantly affect the cell viability in a dosedependent manner (Figure 3(a)). At concentrations of 25 and $50 \mu \mathrm{g} / \mathrm{mL}$ of Te nanowires, the viability was measured to be around $70 \%$ and $45 \%$, respectively. Similarly, the cell death observed at a concentration of $125 \mu \mathrm{g} / \mathrm{mL}$ of Te nanowires was significantly higher than those observed at lower concentrations, and the viability was estimated to be around $9 \%$.

ROS Formation. We carried out a comparative study of intracellular ROS formation in cells treated with various concentrations of Te nanowires for $48 \mathrm{~h}$ (Figure 3(b)). The results showed that the amount of ROS generated in cells treated with $25 \mu \mathrm{g} / \mathrm{mL}$ was slightly higher than that of the controls. However, in comparison to the control cells, cells incubated with 50 and $125 \mu \mathrm{g} / \mathrm{mL}$ of Te nanowires showed significantly higher amounts of ROS formation, indicating that Te nanowires induce ROS formation in the cells. (a)

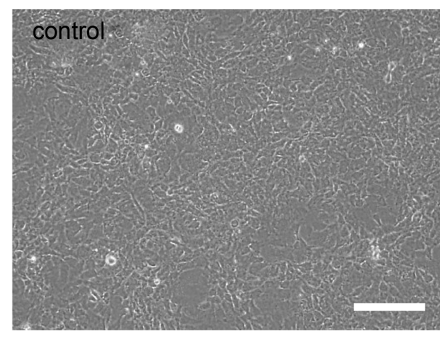

(b)

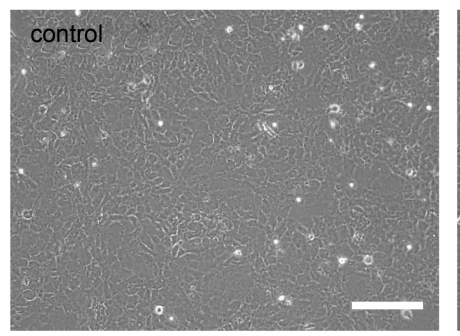

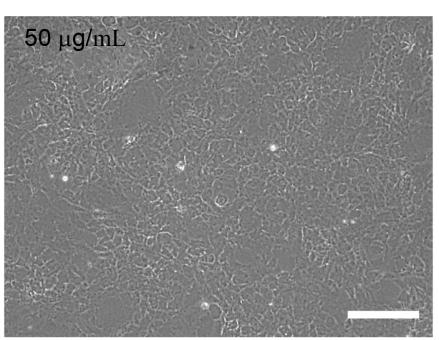

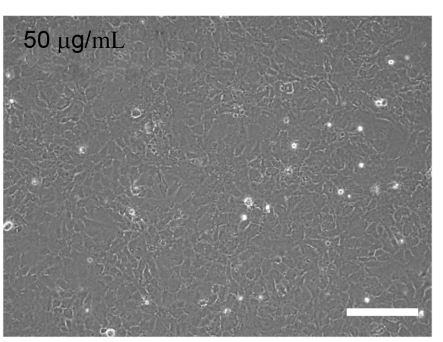

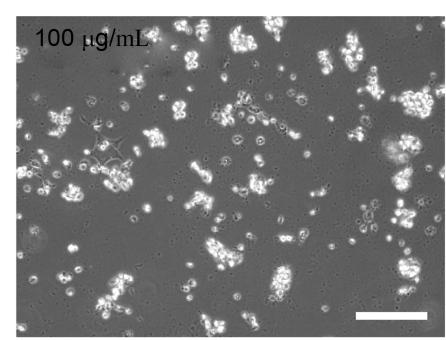

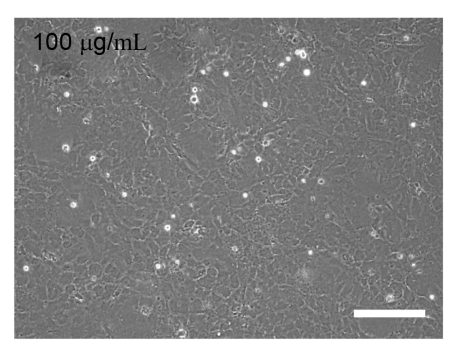

Figure 2. Morphological effects of (a) dodecylbenzenesulfonic acid, sodium salt (SDBS) and (b) poly (sodium 4-styrenesulfonate) (PSS) in BALB/3T3 fibroblast cells after incubation for $48 \mathrm{~h}$. Scale bars: $100 \mu \mathrm{m}$. 

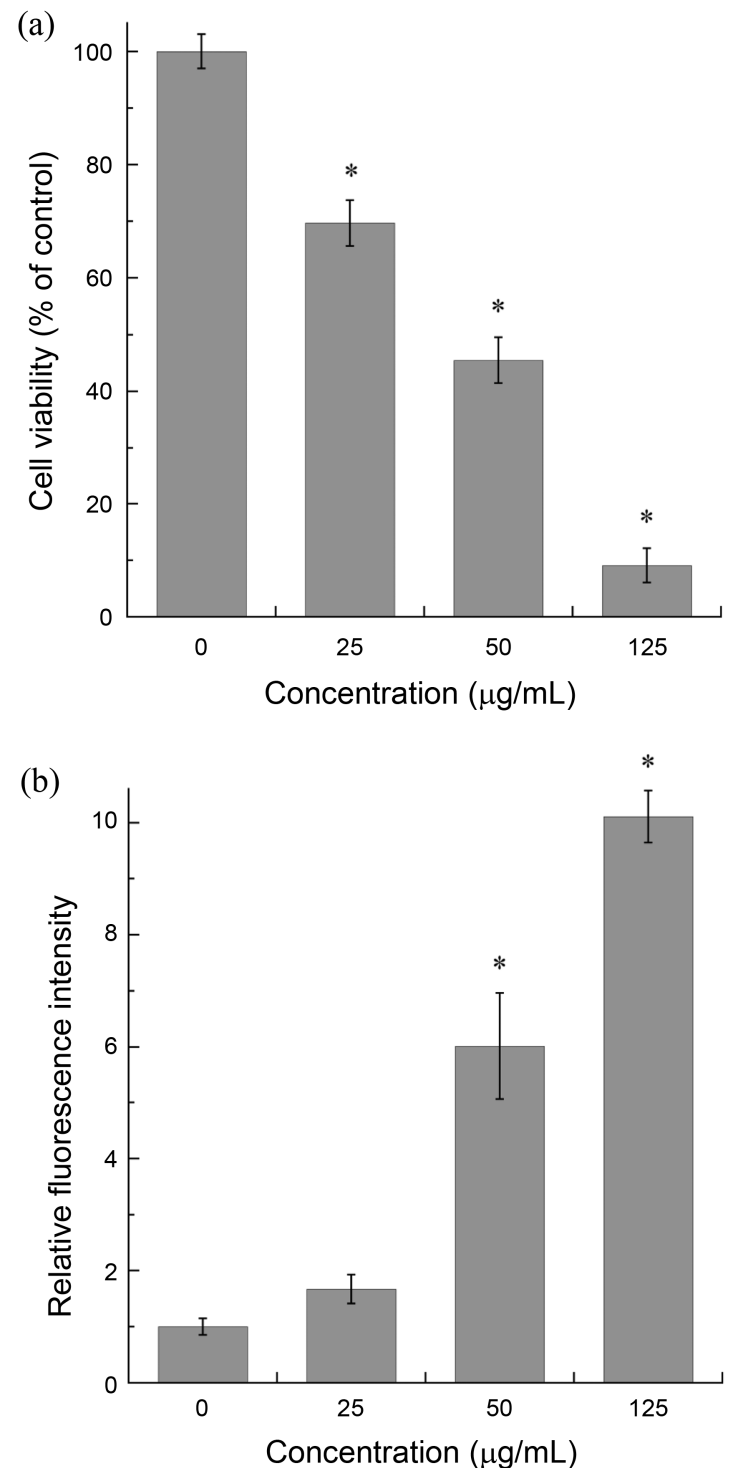

Figure 3. (a) Effects of Te nanowires on cell viability measured by MTT assay. (b) Quantified fluorescent intensity to show relative amount of intracellular ROS formation. Asterisks indicate statistically significant difference from the control; $\mathrm{p}<0.01$.

UV/Vis Absorption Spectra of Te Nanowires. Uniform Te nanowires dispersed in DI water give a deep blue color that is quite visible to the naked eye. The UV/Vis absorption spectrum of the Te nanowires in the present study showed two peaks around $270 \mathrm{~nm}$ and $630 \mathrm{~nm}$ (Figure 4(a), plot 1). These values are close to the reported values for Te nanowires. ${ }^{20-22}$ The first peak at $270 \mathrm{~nm}$ is assumed to result from the allowed transition from the valence band to the conduction band and the second peak at $630 \mathrm{~nm}$ can be assigned to a forbidden direct transition. ${ }^{23}$

We conducted time-dependent observation of absorption spectra of Te nanowires suspended in the DMEM medium alone and the DMEM growth medium separately.

During the performance of cytotoxicity experiments, the UV/Vis spectrum of Te nanowires suspended in a DMEM medium alone for $45 \mathrm{~min}$ showed dramatic changes in color
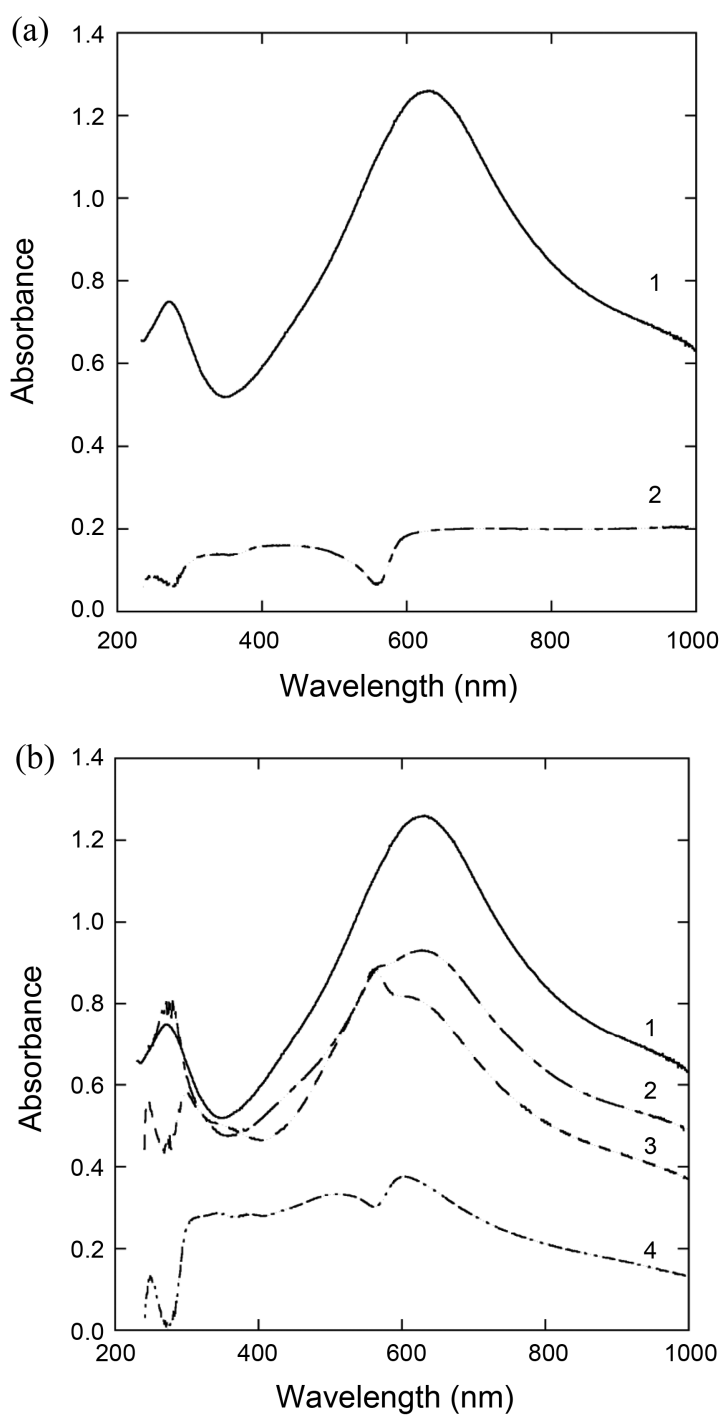

Figure 4. (a) UV/Vis absorption spectra of Te nanowires suspended in (1) water $(50 \mu \mathrm{g} / \mathrm{mL})$, and (2) DMEM medium $(50 \mu \mathrm{g} / \mathrm{mL})$ for $45 \mathrm{~min}$ at $37^{\circ} \mathrm{C}$ inside $\mathrm{CO}_{2}$ incubator. (b) Timedependent changes in UV/Vis absorption spectra of Te nanowires suspended in growth medium (DMEM $+10 \% \mathrm{FBS}+1 \% \mathrm{P} / \mathrm{S})$ at $37{ }^{\circ} \mathrm{C}$ inside a $\mathrm{CO}_{2}$ incubator; (1) $\mathrm{t}=0,(2) \mathrm{t}=45 \mathrm{~min},(\mathbf{3}) \mathrm{t}=24 \mathrm{~h}$, (4) $\mathrm{t}=48 \mathrm{~h}$

and disappearance of the two broad absorption peaks (Figure 4(a), plot 2) with the passage of time and thus, toxicity gradually becomes manifested. In addition, we observed strong aggregation of Te nanowires in the growth medium and subsequent disappearance of blue color, which was readily detectable by the naked eye. However, Te nanowires suspended in the DMEM growth medium showed a gradual change in their absorption spectra with the passage of time (Figure 4(b), plots 1-4). The results indicated that the certain components of the DMEM medium significantly affect the chemical and structural stability of Te nanowires, and further suggest that the physicochemical properties of Te nanowires were significantly altered during exposure of cells.

Zeta Potential and SEM Images of Te Nanowires. The zeta potential for Te nanowires dispersed in water $(50 \mu \mathrm{g} / \mathrm{mL})$ 
(a)

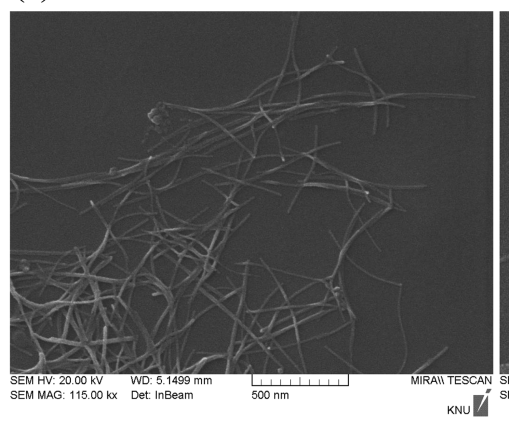

(b)

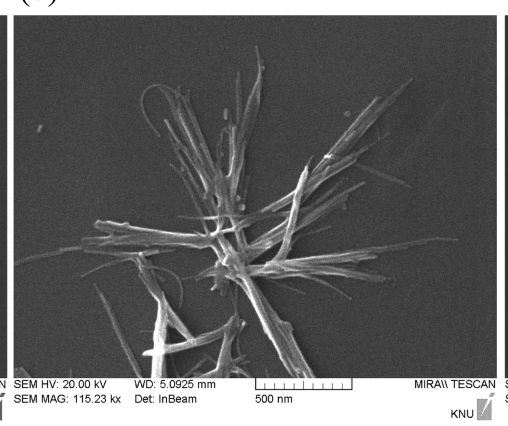

(c)

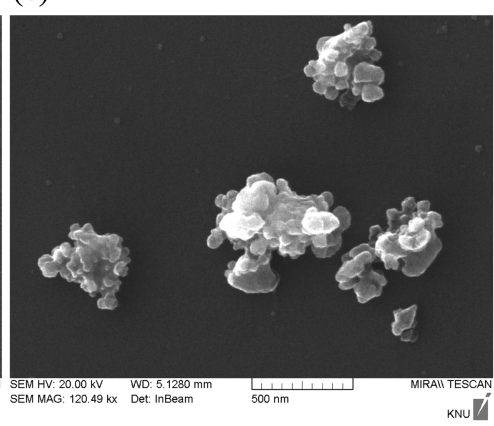

Figure 5. SEM images of Te nanowires suspended in (a) water $(50 \mu \mathrm{g} / \mathrm{mL})$, (b) DMEM medium $(50 \mu \mathrm{g} / \mathrm{mL})$ for $45 \mathrm{~min}$, and (c) growth medium $(50 \mu \mathrm{g} / \mathrm{mL})$ for $48 \mathrm{~h}$ at $37^{\circ} \mathrm{C}$ inside a $\mathrm{CO}_{2}$ incubator. Scale bars: $500 \mathrm{~nm}$.

was highly negative, with an average value of $-37.6 \mathrm{mV}$, indicating that the nanowires were highly stabilized and completely dispersed, having highly negative surface charges. However, the zeta potential of Te nanowires suspended in the DMEM medium $(50 \mu \mathrm{g} / \mathrm{mL})$ alone significantly decreased to $-11.2 \mathrm{mV}$ after $48 \mathrm{~h}$ of incubation, suggesting the desorption of surfactants and subsequent agglomeration of nanowires in the presence of the DMEM medium. These results were also supported by SEM observation of Te nanowires suspended in different types of media. Te nanowires dispersed in water were distributed uniformly and were also highly stabilized (Figure 5(a)). In contrast, Te nanowires suspended in the DMEM medium alone for $45 \mathrm{~min}$ were significantly agglomerated and distorted (Figure 5(b)). Similarly, the nanowires were completely aggregated and nanowire structures were no longer retained when suspended in the DMEM growth medium for $48 \mathrm{~h}$ (Figure 5(c)). These results support the hypothesis that the components of the DMEM medium significantly affect the stability of Te nanowires, and further suggest that the physicochemical properties of Te nanowires were significantly altered during exposure of cells. Also, we measured the total concentration of Te atoms by ICP-AES in the respective concentrations of Te nanowires to estimate the maximum possible concentrations of SDBS and PSS in the given stock solution of nanowires. The results revealed that the given stock solution of nanowires contains $60-80 \%$ of Te atoms and $20-40 \%$ of SDBS and PSS $(1: 1)$. These data primarily indicate that the major constituent of nanowires is tellurium, and it is possibly involved in cytotoxicity of Te nanowires.

No Change in Properties of the Culture Medium. To clarify the issue that the physicochemical properties of the culture medium might have altered and subsequently, led to the death of cells as a result of change in medium and not due to the direct cytotoxic effect of Te itself, we have estimated the relevant parameters, such as $\mathrm{pH}$ and osmolality of various media after incubation of Te nanowires in all the media for selected time-points. The results revealed that the value of $\mathrm{pH}$ and osmolality was almost comparable to those of controls and, thus suggest that the cell death was actually the result of direct cytotoxic effect of Te itself and not due to the change in physicochemical properties of culture medium.
Also, previous studies reported that simple salts containing $\mathrm{TeO}_{3}{ }^{2-}$ (tellurite) and $\mathrm{TeO}_{4}{ }^{2-}$ (tellurate) are toxic to most organisms. ${ }^{24}$ In addition, some diaryl tellurides were found to inhibit the growth of cancer cells (e.g., human MCF-7 breast cancer, HT-29 colon cancer, Colo320 colon cancer). ${ }^{25}$ Based on these observations, we propose that the nontoxic Te atoms $\left(\mathrm{Te}^{0}\right)$ of nanowires might chemically react during incubation with cells in a culture medium and subsequently form toxic moieties (e.g., $\mathrm{Te}^{4+}, \mathrm{Te}^{6+}$, and $\mathrm{Te}^{2-}$ ) of tellurium, leading to cell death. However, the actual mechanism underlying this process of chemical reaction should be investigated further.

Cell Death Mechanism. The electron micrographs showed various aspects of early phase of necrotic cell death events after treatment with Te nanowires (Figure 6). Their organelles were found to be completely disintegrated and the plasma membrane appeared to be totally ruptured. In addition, the mitochondria was damaged and showed various degrees of destruction, from swelling and loss of an electron dense matrix to complete disintegration. Other organelles except the nuclei were not identifiable and appeared to be dilated. The nuclei were observed to be in normal condition and undamaged, although the structures

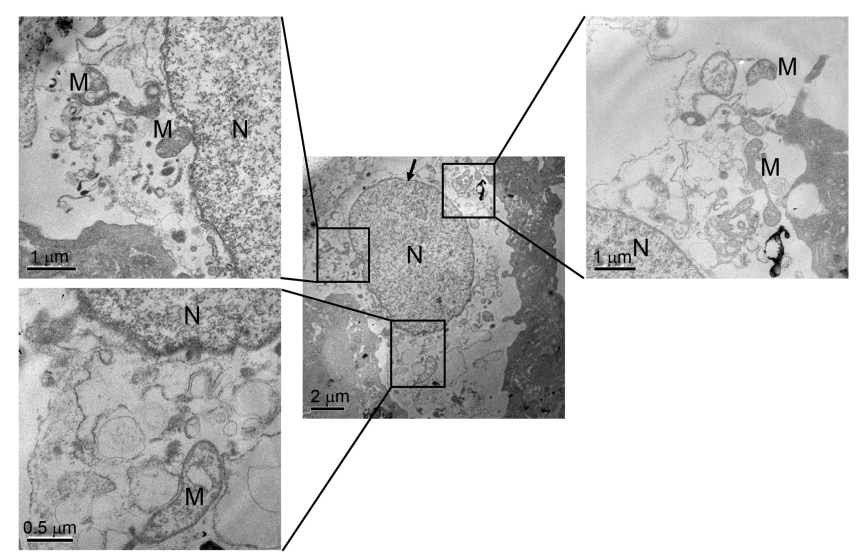

Figure 6. Transmission electron microscopic image of a cell treated with $\mathrm{Te}$ nanowires. Note the characteristic features of necrosis, such as ruptured plasma membrane, damaged mitochondria (M), swollen nucleus $(\mathrm{N})$, and disappearance of other organelles. 
were slightly swollen. The nuclear membranes were well intact with a distinct double layer. These characteristic features indicate that necrosis is the basic pattern of cell death induced by Te nanowires.

\section{Conclusions}

In conclusion, this study analyzed the cytotoxic potential of Te nanowires in BALB/3T3 fibroblast cells. Te nanowires were found to be cytotoxic at all concentrations tested, in a dose-dependent manner. Cells incubated with Te nanowires produced a significant amount of ROS. The UV/Vis spectra of Te nanowires suspended in a culture medium showed drastic changes and disappearance of two broad absorption peaks. SEM images and measurement of zeta potential showed that the components of the DMEM medium significantly affect the chemical and structural stability of Te nanowires, leading to the instability and agglomeration of nanowires in the culture medium. However, the primary reason underlying the instability and gradual degradation of tellurium nanowires in the culture medium should be explored in future study. In addition, TEM images revealed that necrosis was the basic pattern of cell death. This might be caused by the formation of toxic moieties of tellurium, released from nanowire structures, in the bioenvironment. This study should provide initiative to consider potential health and safety risks associated with the use of tellurium nanowires in industry and biological and medical applications.

Acknowledgments. This work was supported by the Priority Research Centers Program through the National Research Foundation of Korea (NRF) funded by the Ministry of Education, Science and Technology (2010-0028289).

\section{References}

1. Oberdörster, G.; Stone, V.; Donaldson, K. Nanotoxicology 2007, 1,
2.

2. Oberdörster, G.; Oberdörster, E.; Oberdörster, J. Environ. Health Perspect. 2005, 113, 823.

3. Lauterwasser, C. Small sizes that matter: opportunities and risks of nanotechnologies, report in co-operation with the OECD International Futures Programme, accessed on 13 November 2008.

4. Donaldson, K.; Stone, V.; Tran, C. L.; Kreyling, W.; Borm, P. J. A. Occup. Environ. Med. 2004, 61, 727.

5. Zheng, R.; Cheng, W.; Wang, E.; Dong, S. Chem. Phys. Lett. 2004, 395, 302.

6. Liu, Z.; Hu, Z.; Xie, Q.; Yang, B.; Wu, J.; Qian, Y. J. Mater. Chem. 2002, 13, 159 .

7. Mayers, B.; Xia, Y. Adv. Mater. 2002, 14, 279.

8. Chen, X.; Wang, Z.; Wang, X.; Wan, J.; Qian, Y. Appl. Phys. A 2005, 80, 1443.

9. Liang, F.; Qian, H. Mater. Chem. Phys. 2009, 113, 523.

10. Liu, L.; Zhang, J.; Su, X.; Mason, R. P. J. Biomed. Nanotechnol. 2008, 4, 524

11. Yu, H.; Gibbons, P. C.; Buhro, W. E. J. Mater. Chem. 2004, 14, 595.

12. Ogra, Y. Anal. Sci. 2009, 25, 1189.

13. Ding, W. J.; Hasegawa, T.; Peng, D.; Hosaka, H.; Seko, Y. J. Trace Elem. Med. Biol. 2002, 6, 99.

14. Deuticke, B.; Lutkemeier, P.; Poser, B. Biochim. Biophys. Acta 1992, 1109, 97.

15. Sailer, B. L.; Liles, N.; Dickerson, S.; Chasteen, T. G. Arch. Toxicol. 2003, 77, 30.

16. Hartung, T.; Bremer, S.; Casati, S.; Coecke, S.; Corvi, R.; Fortaner, S.; Gribaldo, L.; Halder, M.; Roi, A. J.; Prieto, P.; Sabbioni, E.; Worth, A.; Zuang, V. Altern. Lab. Anim. 2003, 31, 473.

17. Mazzotti, F.; Sabbioni, E.; Ghiani, M.; Cocco, B.; Ceccatelli, R.; Fortaner, S. Altern. Lab. Anim. 2001, 29, 601.

18. Ponti, J.; Sabbioni, E.; Munaro, B.; Broggi, F.; Marmorato, P.; Franchini, F.; Colognato, R.; Rossi, F. Mutagen. 2009, 24, 439.

19. Vinod, T. P.; Yang, M.; Kim, J.; Kotov, N. A. Langmuir 2009, 25, 13545.

20. Gautam, U. K.; Rao, C. N. R. J. Mater. Chem. 2004, 14, 2530.

21. Liu, H.; Liu, S.; Huang, K. Mater. Lett. 2008, 62, 1983.

22. Qian, H. S.; Yu, S. H.; Gong, J. Y.; Luo, L. B.; Fei, L. F. Langmuir 2006, 22, 3830 .

23. Fendler, J. H. Chem. Mater. 1996, 8, 1616.

24. Cunha, R. L. O. R.; Gouvea, I. E.; Juliano, L. An. Acad. Bras. Ciênc. 2009, 81, 393

25. Chasteen, T. G.; Bentley, R. Chem. Rev. 2003, 103, 1. 